\title{
BMJ Open Association of the incidence of atopic dermatitis until 3 years old with birth month and with sunshine duration and humidity in the first 6 months of life: Japan Environment and Children's Study
}

Hiroshi Yokomichi (D) , ${ }^{1}$ Mie Mochizuki, ${ }^{2}$ Akiko Tsuchida, ${ }^{3}$ Reiji Kojima, ${ }^{1}$ Sayaka Horiuchi, ${ }^{4}$ Tadao Ooka, ${ }^{1}$ Yuka Akiyama, ${ }^{1}$ Kunio Miyake, ${ }^{1}$ Sanae Otawa, ${ }^{4}$ Ryoji Shinohara, ${ }^{4}$ Hidekuni Inadera, ${ }^{3}$ Zentaro Yamagata, ${ }^{1,4}$ Japan Environment and Children's Study Group 5

To cite: Yokomichi H, Mochizuki M, Tsuchida A, et al. Association of the incidence of atopic dermatitis until 3 years old with birth month and with sunshine duration and humidity in the first 6 months of life: Japan Environment and Children's Study. BMJ Open 2021;11:e047226. doi:10.1136/ bmjopen-2020-047226

- Prepublication history and additional online supplemental material for this paper are available online. To view these files, please visit the journal online. To view these files, please visit the journal online (http://dx.doi.org/10.1136/ bmjopen-2020-047226).

Received 23 November 2020 Accepted 08 June 2021

Check for updates

(C) Author(s) (or their employer(s)) 2021. Re-use permitted under CC BY-NC. No commercial re-use. See rights and permissions. Published by BMJ.

For numbered affiliations see end of article.

Correspondence to Dr Hiroshi Yokomichi; hyokomichi@yamanashi.ac.jp

\section{ABSTRACT}

Objective To compare the incidence of atopic dermatitis in children aged from 6 months to 3 years across birth seasons and climate conditions.

Design Cohort study.

Setting Fifteen regional centres across Japan.

Participants A total of 100304 children born from 2011 to 2014.

Exposure Birth month, and mean sunshine duration (short/long) and humidity (high/low) in the first 6 months of life.

Primary outcome measure Incidence of atopic dermatitis.

Results The highest incidence of atopic dermatitis was in children born in the months of October to December. The lowest incidence of atopic dermatitis was in the months of April to June and in periods with a long duration of sunshine and high humidity. Low humidity was significantly associated with a higher incidence of atopic dermatitis. However, this significant difference disappeared when the birth season and parental history of allergic disease were considered in multivariate analysis.

Conclusions In Japan, being born in the late autumn to early winter months is associated with a risk of developing atopic dermatitis until the age of 3 years. Sunshine duration and humidity from birth to 6 months of age are not associated with the incidence of atopic dermatitis.

\section{INTRODUCTION}

Approximately $10 \%-20 \%$ of children have atopic dermatitis (AD). ${ }^{12}$ Many genetic and environmental factors may be associated with the incidence and aggravation of $\mathrm{AD} .^{3}$ An epidemiological investigation identifying factors associated with $\mathrm{AD}$ would be helpful for reducing the number of child patients who suffer from this disease from infancy.

\section{Strengths and limitations of this study}

Six-monthly meteorological data were used, corresponding to individual children.

- The parental history of allergic disease was considered.

- A history of bacterial infection was not considered theoretically as a confounder.

- Metrological data of children's residence were not individual but averaged.

The severity of atopic dermatitis was not analysed.

Possible environmental factors of $\mathrm{AD}$ include seasonal climate conditions, chemical irritants, bacterial colonisation, psychological stress ${ }^{4}$ and birth month. ${ }^{4}$ Among environmental factors, being born from autumn to winter in the northern hemisphere increases the risk of developing $\mathrm{AD}$, while birth from spring to summer may decrease the incidence of $\mathrm{AD} .^{5}$ The mechanism of this association could be confounded by prevailing seasonal viruses, airborne natural antigens, or sunshine duration and humidity, which could be related to psychological stress, dry skin, and itchiness. Exposure to ultraviolet may improve skin barrier performance, ${ }^{6}$ and may reduce the risk of developing $\mathrm{AD} .^{7}$ Low humidity may be related to a high incidence of $\mathrm{AD}^{8}$ Relating birth cohort data ${ }^{9-12}$ and metrological data ${ }^{13}$ may help answer the question of how birth month is associated with the incidence of $\mathrm{AD}$. Therefore, this study aimed to investigate how birth month is associated with the incidence of $\mathrm{AD}$. 


\section{METHODS}

\section{Measures}

Details on the Japan Environment and Children's Study (JECS) project are published elsewhere. ${ }^{9}$ Approximately 100000 expecting mothers who lived in designated study areas were recruited over 3 years from January 2011. Participating children were followed until they reached 13 years old. Exposure to environmental factors was assessed by chemical analyses of biospecimens (blood, cord blood, urine, breast milk and hair), household environment measurements and computational simulations using monitoring data and questionnaires. One of the JECS' priority outcomes was immune system disorders (allergic diseases). ${ }^{9}$ The JECS comprises a cohort of 104062 children born from 2011 to 2014 in 15 Regional Centres covering 18 prefectures across Japan. ${ }^{14}$ We used the JECS data 'jecs-ta-20190930-qsn' for generating questionnaires, which were sent to caregivers by post when their children were aged 6 months, 1 year, 2 years and 3 years. We asked if physicians had diagnosed the children with $\mathrm{AD}$.

In Japan, sunshine duration almost peaks on 21 June (the summer solstice) and is at its shortest on 21 December (the winter solstice). Humidity, which is lowest in winter, rises proportionally with a rise in sunshine duration to summer. Sunshine duration varies depending on regions from approximately 125-180 hours/month. Humidity also varies depending on region and month from approximately $50 \%-80 \%$ in Japan. Therefore, sunshine duration and humidity of the 18 studied prefectures from the northern to southern regions of Japan varied. Overall in Japan, among seasons, summer has the longest sunshine duration and highest humidity, and winter has the shortest sunshine duration and lowest humidity.

We collected climate condition data per prefecture and month from the Japan Meteorological Agency website. ${ }^{13}$ The sunshine duration was defined as hours with $\geq 120$ Watt $/ \mathrm{m}^{2}$ of direct sunshine, as measured by a solarimeter. The mean sunshine duration and humidity of the 18 prefectures from April 2011 to March 2015 were 166.1 (SD: 45.4) hours/month and 68.4\% (SD: 7.6\%), respectively. These values were used as cut-off values for categorising children as being exposed to a long/short sunshine duration and high/low humidity while taking into account their resident area and birth month. For this categorisation, the mean sunshine duration and humidity in the child's resident area over 6 months beginning with the child's birth month were compared with the cut-off values. An example of this categorisation is that a child who was born in April 2011 would be categorised on the basis of meteorological data from April to September 2011.

For considering a child's genetic predisposition to AD, we used a parental history of asthma, allergic rhinitis, pollen allergy, $\mathrm{AD}$, allergic conjunctivitis and/or food allergy. The father and mother were asked individually about a history of allergic diseases to determine their experience of each diagnosed disease by a physician.

\section{Analyses}

The 12 months of the year were categorised into four seasons in three different ways (ie, starting with January to March, February to April or March to May). We compared Kaplan-Meier curves of the incidence of $\mathrm{AD}$ among seasons by performing log-rank tests. The incidence of $\mathrm{AD}$ by birth month and season until 3 years of age was calculated. We also compared the incidence of $\mathrm{AD}$ using Kaplan-Meier curves for children who were born in regions with long versus short sunshine, with high versus low humidity and with a long/short sunshine duration and high/low humidity. Because we found a difference in the incidence of $\mathrm{AD}$ among birth seasons, we compared this incidence among seasons by strata of children whose parents had a history of allergic disease and those who did not have a history of allergic disease. We performed Cox regression by birth season, humidity, and parental history of allergy, which were considered to be risk factors of AD from Kaplan-Meier curves. Data from participants who were lost to follow-up or developed $\mathrm{AD}$ after 3 years of age were considered as being censored. We conducted all statistical analyses using SAS statistical software V.9.4 (SAS Institute). Kaplan-Meier curves were drawn using RStudio V.1.2.1335 (R Project for Statistical Computing, Vienna, Austria). Two-sided $p$ values of $<0.05$ were considered to indicate a significant difference.

\section{Patient and public involvement}

Written informed consent was obtained from all participants. Patient consent for publication was not required. Patients or the public were not involved in the design, conduct, reporting, or dissemination plans of our research.

\section{RESULTS}

The number of participants who answered the questionnaire decreased with the child's age. By the ages of 6 months, 1 year, 2 years and 3 years, 1715 of 100304 children (response rate: 96.4\%), 4505 of 90549 children (response rate: $87.0 \%$ ), 7299 of 84859 children (response rate: $81.5 \%$ ), and 9704 of 80176 children (response rate for $77.0 \%$ ), respectively, had developed AD. Online supplemental table 1 shows the characteristics of the participants. The highest and lowest incidence of $\mathrm{AD}$ was observed in the October to December group and the April to June group, respectively (figure 1). The overall incidence per 100 person-years varied from 5.27 (October to December) to 4.31 (April to June) (online supplemental table 1). By month, the highest incidence was in children born in October and December (online supplemental figure 1). Online supplemental figures 2 and 3 show the results after varying how the months were grouped. The order of accumulated incidence of $\mathrm{AD}$ among the seasons did not change much from 6 months to 3 years old.

We also assessed the incidence of $\mathrm{AD}$ among four climate categories, including combinations of a long/ 
Birth month and atopic dermatitis incidence

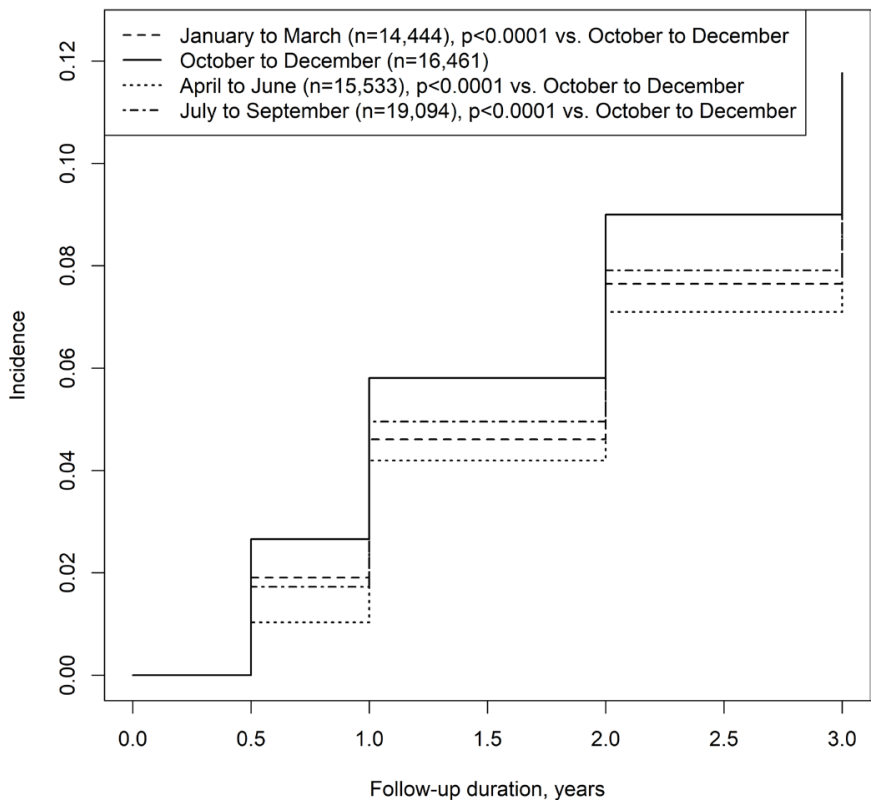

Figure 1 Birth season and incidence of atopic dermatitis in a Japanese birth cohort with the seasons starting from January.

short sunshine duration and high/low humidity, in the first 6 months of life. High or low sunshine duration was not associated with the incidence of $\mathrm{AD}$ (figure 2), while low humidity was associated with the incidence of AD compared with high humidity (figure 3). Among the long/short sunshine duration and high/low humidity groups, the short sunshine duration and low humidity group had a significantly higher incidence of AD than the long sunshine duration and high humidity group (figure 4).

Sunshine duration 6 mo from birth and $A D$ incidence

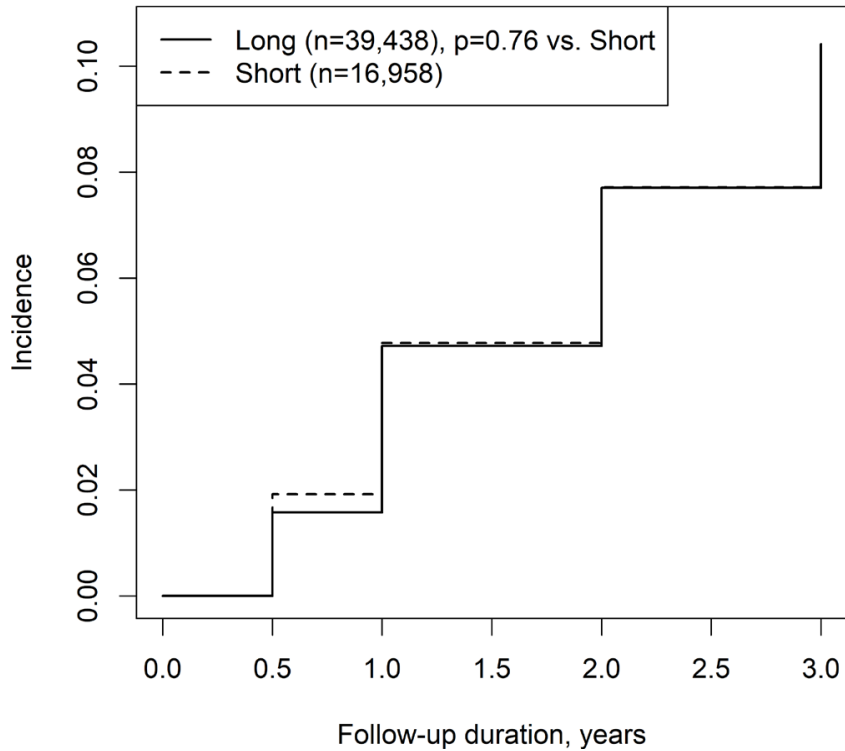

Figure 2 Incidence of atopic dermatitis in regions with a long/short mean sunshine duration.
Humidity 6 mo from birth and atopic dermatitis incidence

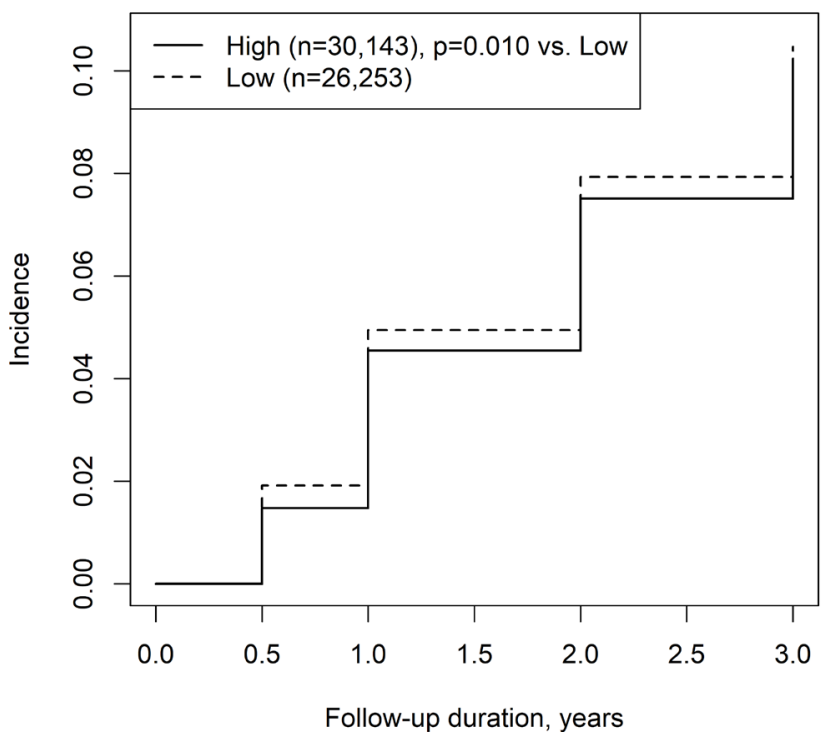

Figure 3 Incidence of atopic dermatitis in regions with a high/low mean humidity.

We also analysed the incidence of $\mathrm{AD}$ based on parental history of any allergic disease. Online supplemental figures 4 and 5 show the incidence of $\mathrm{AD}$ by birth season, as stratified by parental history of allergic disease. The observed highest and lowest incidence of $\mathrm{AD}$ shown in online supplemental figures 4 and 5 was consistent with that shown in figure 1.

Table 1 shows the results of Cox proportional regression for risk factors of the incidence of AD. The adjusted HR showed that birth between October and December

Sunshine \& humidity vs. atopic dermatitis incidence

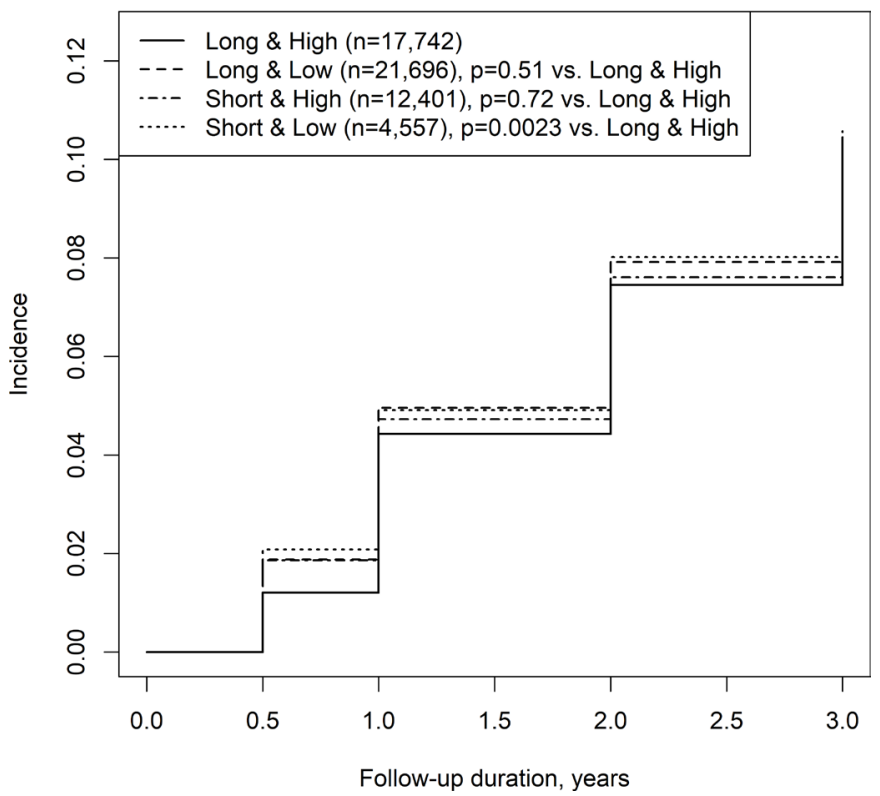

Figure 4 Incidence of atopic dermatitis in regions with a long/short mean sunshine duration and high/low mean humidity. 
Table 1 HRs (95\% Cls) of possible factors involved in the incidence of atopic dermatitis until 3 years old

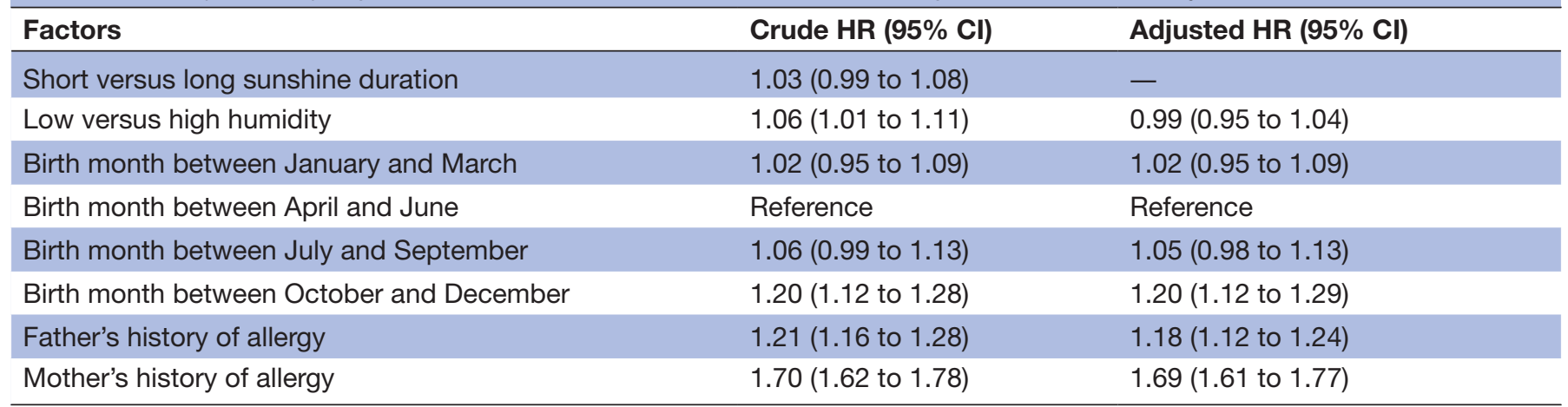

and the father's and mother's history of allergy were risk factors of $\mathrm{AD}$ until the age of 3 years in the child.

\section{DISCUSSION}

The current study showed that the incidence of AD until the age of 3 years was highest when children were born between October and December and lowest when they were born between April and June. Consideration of parental history of allergic disease did not alter this association. Multivariate analysis showed that sunshine duration or humidity was not associated with the incidence of AD.

Potential aetiologies of this association are bacterial infection, dry air, high solar radiation/sunshine hours and atmospheric pressure. Dry skin and itchiness, which develop into $\mathrm{AD}$, are common in low humidity. In the USA, a higher incidence of AD is associated with indoor heating use and low humidity, ultraviolet exposure and outdoor temperature. ${ }^{15}$ In Japan, a negative correlation between humidity and dermatological visits concerning AD was observed. ${ }^{16}$ In our study, we did not observe a clear correlation between high or low humidity in the first 6 months of life and the risk of $\mathrm{AD}$, which suggested that humidity is not critical for inducing AD.

In Japan, $\mathrm{AD}$ shows remittance in $50 \%$ of 4 -month-old patients before the age of 18 months. However, a previous study reported a high cumulative incidence rate of $\mathrm{AD}$ of $30 \%$ in children who were younger than 3 years. ${ }^{17}$ In mice, low humidity caused a higher cutaneous immune reaction by an increase in Langerhans cells and penetration of allergen. ${ }^{18}$ The mean humidity in Japan was $70 \%$ in 2016 , which is not high compared with other countries. ${ }^{19}$ High humidity is clearly associated with a higher incidence of hand, foot and mouth disease with fever, ulcers and vesicles. ${ }^{20}$ Dry and itchy skin are caused by low humidity and low temperature. ${ }^{8}$ However, in this study, there was no effect of high or low humidity on the risk of $\mathrm{AD}$ when birth month and parental history of allergic disease were considered (table 1). Therefore, in the present children, humidity did not appear to be important for inducing AD. Our study also did not suggest that a long sunshine duration induces AD.
Ultraviolet lighting is sometimes used clinically for reducing itchiness from severe $\mathrm{AD} .^{21}$ However, a long sunshine duration can also cause dry skin, which is a risk factor of AD. Furthermore, heat from a long sunshine duration can cause sweating, leading to itchiness and psychological stress from discomfort, both of which can exacerbate $\mathrm{AD}$. In Japan, humidity is fairly constant. Therefore, we initially suspected that in Japan, itchiness and psychological stress from a long duration of sunshine may be a cause of AD in children. However, in contrast to our speculation, a combination of short sunshine duration and low humidity was associated with the highest incidence of $\mathrm{AD}$ (figure 4).

Notably, the association between the incidence rate of $\mathrm{AD}$ across birth seasons, sunshine duration, and humidity levels hardly changed from 6 months to 3 years old (figures 1 and 4 ). This finding indicates that environmental factors with an adverse or preventive effect persist through early childhood. Allergic predisposition may not be determined until 6 months of age. Before that age, most infants only drink milk and do not eat allergenic solid products. A meta-analysis did not show clear evidence for a protective effect of breast feeding on the incidence of $\mathrm{AD}{ }^{22}$ Our analysis did not examine feeding behaviour of the children.

Confounding factors of our analysis need to be considered. When we considered genetic predisposition, we found a high incidence of AD in children who were born in October to December, regardless of whether their parents had allergic diseases (figure 1, online supplemental figures 4, 5 and table 1 ). Because parents do not plan the birth month of a newborn with a genetic predisposition to $\mathrm{AD}$, this factor could not have theoretically confounded the observed associations.

Serum vitamin D levels may be involved in the association between birth month and the incidence of $\mathrm{AD}$. Maternal vitamin D supplementation does not affect the risk of $\mathrm{AD}$ at 3 years old. ${ }^{23}$ However, in an Australian study, a far distance from the equator was associated with a higher prevalence of eczema. ${ }^{24}$ Ultraviolet B irradiation decreases inflammation and promotes skin barrier function. ${ }^{6}$ Our data showed that birth between April and June was the most protective period against the incidence of 
$\mathrm{AD}$. A high or low prevalence of $\mathrm{AD}$ among birth seasons was preserved from 6 months to 3 years old.

\section{Limitations}

Our study has the following limitations. First, our results are limited by infection not being examined as a potential confounder. Second, we used 6 monthly means of sunshine duration and humidity. Individual experience was not considered. Third, dichotomisations of a long/ short sunshine duration and high/low humidity were determined by single cut-off values. Fourth, the incidence of $\mathrm{AD}$ was reported by caregivers based on the physician's diagnosis. There could have been recall bias of caregivers. Fifth, physicians who diagnosed children included specialists of $\mathrm{AD}$ and non-specialists. Physicians might have underdiagnosed $\mathrm{AD}$ in infancy because this diagnosis can cause stigma to children and caregivers. ${ }^{25}$ Sixth, we were unable to consider details of AD. Because $\mathrm{AD}$ can be caused by several mechanisms, analysis of disease severity may lead to a further understanding of its aetiology.

\section{CONCLUSIONS}

Births in October to December have the highest incidence of $\mathrm{AD}$ until the age of 3 years. This result is consistent when a parental history of allergic disease is considered. A high or low incidence of $\mathrm{AD}$ by birth season persists from 6 months through to 3 years in childhood. Although the lowest incidence of $\mathrm{AD}$ is found in residents in regions with a long sunshine duration and high humidity in crude data, multivariate analysis shows no association of sunshine duration and humidity with the incidence of $\mathrm{AD}$.

\section{Author affiliations}

${ }^{1}$ Department of Health Sciences, University of Yamanashi - Graduate School of Medical Science Campus, Chuo, Japan

${ }^{2}$ Department of Paediatrics, University of Yamanashi - Graduate School of Medical Science Campus, Chuo, Japan

${ }^{3}$ Department of Public Health, University of Toyama, Toyama, Japan

${ }^{4}$ Centre for Birth Cohort Studies, University of Yamanashi - Graduate School of

Medical Science Campus, Chuo, Japan

${ }^{5} J a p a n$ Environment and Children's Study, Tokyo, Japan

Acknowledgements We thank Dr Ellen Knapp for editing a draft of this manuscript. †Members of the JECS Group as of 2020: Michihiro Kamijima (principal investigator, Nagoya City University, Nagoya, Japan), Shin Yamazaki (National Institute for Environmental Studies, Tsukuba, Japan), Yukihiro Ohya (National Center for Child Health and Development, Tokyo, Japan), Reiko Kishi (Hokkaido University, Sapporo, Japan), Nobuo Yaegashi (Tohoku University, Sendai, Japan), Koichi Hashimoto (Fukushima Medical University, Fukushima, Japan), Chisato Mori (Chiba University, Chiba, Japan), Shuichi Ito (Yokohama City University, Yokohama, Japan), Zentaro Yamagata (University of Yamanashi, Chuo, Japan), Hidekuni Inadera (University of Toyama, Toyama, Japan), Takeo Nakayama (Kyoto University, Kyoto, Japan), Hiroyasu Iso (Osaka University, Suita, Japan), Masayuki Shima (Hyogo College of Medicine, Nishinomiya, Japan), Youichi Kurozawa (Tottori University, Yonago, Japan), Narufumi Suganuma (Kochi University, Nankoku, Japan), Koichi Kusuhara (University of Occupational and Environmental Health, Kitakyushu, Japan), and Takahiko Katoh (Kumamoto University, Kumamoto, Japan).

Contributors All the authors agreed with the manuscript's results and conclusion and approved the final version of the manuscript. HY and MM conceived the study. $\mathrm{HY}$ and MM contributed to the design of the study and interpretation of the data analyses. $\mathrm{HY}$ analysed the data. $\mathrm{HY}, \mathrm{MM}, \mathrm{AT}, \mathrm{RK}, \mathrm{SH}, \mathrm{TO}, \mathrm{YA}$ and $\mathrm{KM}$ wrote the first draft of the manuscript. SH, SO, RS, HI and ZY contributed to data collection. All authors contributed to revision of the manuscript. ZY was responsible for data integrity. $Z Y$ obtained funding.

Funding This work was supported by the Ministry of the Environment, Japan. Competing interests None declared.

Patient consent for publication Not required.

Ethics approval The protocol was approved by the Ministry of the Environment's Institutional Review Board on Epidemiological Studies (no. 100910001) and by the Ethics Committees of all participating institutions, in accordance with the ethical guidelines and regulations of the Declaration of Helsinki.

Provenance and peer review Not commissioned; externally peer reviewed.

Data availability statement Data are available on reasonable request. Data are unsuitable for public deposition due to ethical restrictions and legal framework of Japan. It is prohibited by the Act on the Protection of Personal Information (Act No. 57 of 30 May 2003, amendment on 9 September 2015) to publicly deposit the data containing personal information. Ethical Guidelines for Medical and Health Research Involving Human Subjects enforced by the Japan Ministry of Education, Culture, Sports, Science and Technology and the Ministry of Health, Labour and Welfare also restricts the open sharing of the epidemiologic data. All inquiries about access to data should be sent to: jecs-en@nies.go.jp. The person responsible for handling enquiries sent to this email address is Dr Shoji F. Nakayama, JECS Programme Office, National Institute for Environmental Studies.

Supplemental material This content has been supplied by the author(s). It has not been vetted by BMJ Publishing Group Limited (BMJ) and may not have been peer-reviewed. Any opinions or recommendations discussed are solely those of the author(s) and are not endorsed by BMJ. BMJ disclaims all liability and responsibility arising from any reliance placed on the content. Where the content includes any translated material, BMJ does not warrant the accuracy and reliability of the translations (including but not limited to local regulations, clinical guidelines, terminology, drug names and drug dosages), and is not responsible for any error and/or omissions arising from translation and adaptation or otherwise.

Open access This is an open access article distributed in accordance with the Creative Commons Attribution Non Commercial (CC BY-NC 4.0) license, which permits others to distribute, remix, adapt, build upon this work non-commercially, and license their derivative works on different terms, provided the original work is properly cited, appropriate credit is given, any changes made indicated, and the use is non-commercial. See: http://creativecommons.org/licenses/by-nc/4.0/.

ORCID iD

Hiroshi Yokomichi http://orcid.org/0000-0001-7369-155X

\section{REFERENCES}

1 Deckers IAG, McLean S, Linssen S, et al. Investigating international time trends in the incidence and prevalence of atopic eczema 19902010: a systematic review of epidemiological studies. PLoS One 2012;7:e39803.

2 Weidinger S, Novak N. Atopic dermatitis. Lancet 2016;387:1109-22.

3 Katayama I, Aihara M, Ohya Y, et al. Japanese guidelines for atopic dermatitis 2017. Allergol Int 2017;66:230-47.

4 Kusunoki T, Asai K, Harazaki M, et al. Month of birth and prevalence of atopic dermatitis in schoolchildren: dry skin in early infancy as a possible etiologic factor. J Allergy Clin Immunol 1999;103:1148-52.

5 Calov M, Alinaghi F, Hamann CR, et al. The association between season of birth and atopic dermatitis in the Northern Hemisphere: a systematic review and meta-analysis. J Allergy Clin Immunol Pract 2020;8:674-80.

6 Thyssen JP, Zirwas MJ, Elias PM. Potential role of reduced environmental UV exposure as a driver of the current epidemic of atopic dermatitis. J Allergy Clin Immunol 2015;136:1163-9.

7 Rueter K, Jones AP, Siafarikas A, et al. Direct infant UV light exposure is associated with eczema and immune development. $J$ Allergy Clin Immunol 2019;143:1012-20.

8 Engebretsen KA, Johansen JD, Kezic S, et al. The effect of environmental humidity and temperature on skin barrier function and dermatitis. J Eur Acad Dermatol Venereol 2016;30:223-49.

9 Kawamoto T, Nitta H, Murata K, et al. Rationale and study design of the Japan environment and children's study (JECS). BMC Public Health 2014;14:25. 
10 Michikawa T, Nitta H, Nakayama SF, et al. Baseline profile of participants in the Japan environment and children's study (JECS). $J$ Epidemiol 2018;28:99-104.

11 Yokomichi $\mathrm{H}$, Kojima R, Horiuchi S, et al. Effectiveness of influenza vaccination in infants and toddlers with and without prior infection history: the Japan environment and children's study. Vaccine 2021;39:1800-4.

12 Kojima R, Yokomichi H, Akiyama Y, et al. Association between preterm birth and maternal allergy considering IgE level. Pediatr Int 2021. doi:10.1111/ped.14635. [Epub ahead of print: 04 Feb 2021].

13 Ministry of Internal Affairs and Communications. Statistics of Japan 2018, 2018. Available: https://www.e-stat.go.jp/en

14 Iwai-Shimada M, Nakayama SF, Isobe T, et al. Questionnaire results on exposure characteristics of pregnant women participating in the Japan environment and children study (JECS). Environ Health Prev Med 2018;23:45

15 Silverberg Jl, Hanifin J, Simpson EL. Climatic factors are associated with childhood eczema prevalence in the United States. J Invest Dermatol 2013;133:1752-9.

16 Furue M, Yamazaki S, Jimbow K, et al. Prevalence of dermatological disorders in Japan: a nationwide, cross-sectional, seasonal, multicenter, hospital-based study. J Dermatol 2011;38:310-20.

17 Kohno Y. Identification of causative and exacerbation factors of atopic dermatitis and studies for improvment of living environment to prevent the development and exacerbation of symptoms. Reports of research on allegic disease and immunology by Ministry of Health, Labour and Wefare of Japan 2006-2007 2008:173-7.

18 Hosoi J, Hariya T, Denda M, et al. Regulation of the cutaneous allergic reaction by humidity. Contact Dermatitis 2000;42:81-4.

19 El Dorado Weather, Inc. Current world humidity, 2020. Available: https://www.eldoradoweather.com/forecast/world-forecasts/worldhumidity.html

20 Onozuka D, Hashizume M. The influence of temperature and humidity on the incidence of hand, foot, and mouth disease in Japan. Sci Total Environ 2011;410-411:119-25.

21 Hoare C, Li Wan Po A, Williams H. Systematic review of treatments for atopic eczema. Health Technol Assess 2000;4:1-191.

22 Yang YW, Tsai CL, Lu CY. Exclusive breastfeeding and incident atopic dermatitis in childhood: a systematic review and meta-analysis of prospective cohort studies. Br J Dermatol 2009;161:373-83.

23 Chawes BL, Bønnelykke K, Stokholm J, et al. Effect of vitamin D3 supplementation during pregnancy on risk of persistent wheeze in the offspring: a randomized clinical trial. JAMA 2016;315:353-61.

24 Osborne NJ, Ukoumunne OC, Wake M, et al. Prevalence of eczema and food allergy is associated with latitude in Australia. J Allergy Clin Immunol 2012;129:865-7.

25 Chernyshov PV. Stigmatization and self-perception in children with atopic dermatitis. Clin Cosmet Investig Dermatol 2016;9:159-66. 